> La neurorétine est une unité fonctionnelle du système nerveux central assurant la conversion d'un signal lumineux en un influx nerveux. D'origine neuroectodermique, dérivée du diencéphale, la neurorétine est un tissu stratifié, composé de six types de cellules neuronales (deux types de photorécepteurs : les cônes et les bâtonnets; les cellules horizontales, bipolaires, amacrines et ganglionnaires) et de trois types de cellules gliales (les cellules gliales de Müller, les astrocytes et les cellules microgliales). La neurorétine repose sur l'épithélium pigmentaire rétinien, l'ensemble constituant la rétine. L'existence des barrières hémato-rétiniennes interne et externe et des jonctions intra-rétiniennes rend compte de la finesse de la régulation des échanges de la rétine avec la circulation et au sein de la rétine ellemême. La zone centrale de la rétine humaine, la macula, zone hautement spécialisée pour assurer l'acuité visuelle, présente des spécificités anatomiques. Les méthodes d'imagerie récentes permettent d'enrichir nos connaissances sur les caractéristiques anatomiques et fonctionnelles de la rétine, qui restent encore imparfaitement décrites. <

\section{Anatomie macroscopique}

La rétine tapisse le fond de l'œil (Figure I A). C'est une fine tunique transparente, de moins de $500 \mu \mathrm{m}$ in vivo, laissant apparaître la vascularisation des pigments et la vascularisation de la choroïde, à l'origine de l'aspect rose-orangé du fond d'œil (Figure I A, B). La tête du nerf optique est visible macroscopiquement sous forme d'un disque de couleur rosée, plus claire que celle de la rétine, autour duquel émergent des artères et des veines rétiniennes, appelé papille optique. La macula est une zone de la rétine postérieure dont le centre est avasculaire.

Vignette (Photo (c) Inserm - Marion Vincent).

\section{Rétine (1)}

Une série animée par Thierry Jouault

Anatomie de la rétine

Francine Behar-Cohen ${ }^{1-3}$, Emmanuelle Gelizé ${ }^{2}$, Laurent Jonet ${ }^{2}$, Patricia Lassiaz ${ }^{2}$

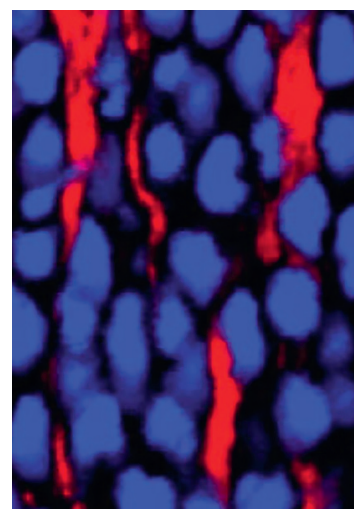

IOphtalmopole, Hôpital Cochin, 27 rue du Faubourg SaintJacques, 75014 Paris, France. ${ }^{2}$ Centre de recherches des cordeliers; Inserm UMR 1138, Physiopathologie des maladies oculaires : innovations thérapeutiques ; Université de Paris ; 15 rue de l'École de Médecine, 75006 Paris, France.

${ }^{3}$ Université de Paris, France. francine.behar@inserm.com

Elle est discernable à l'examen du fond d'œil par sa coloration orangée due à la présence des pigments xanthophylles ${ }^{1}$. Elle est située en temporal de la papille optique. La fovéa, au centre de la macula, constituée uniquement de cônes, permet l'acuité visuelle. La rétine s'étend de l'ora serrata, en avant, jusqu'au nerf optique, au pôle postérieur du globe oculaire (Figure I A). La pars plana, partie la plus antérieure de la rétine, non décollable, est la région dans laquelle sont introduits les instruments de chirurgie vitréo-rétinienne et où sont réalisées les injections intravitréennes (Figure 1 A). Le diamètre transverse interne de l'œil emmétrope ${ }^{2}$ adulte est d'environ $22 \mathrm{~mm}$. La rétine couvre environ 15 à $16 \mathrm{~mm}$ du diamètre interne de l'œil, et $72 \%$ de la surface de la sphère oculaire. La sclère, coque blanche qui assure la rigidité au globe, mesure moins d'un millimètre d'épaisseur.

\section{Vascularisation de la rétine : vaisseaux rétiniens et choroïdiens}

La rétine est vascularisée par deux systèmes vasculaires différents, sans connexions anatomiques en conditions physiologiques: le réseau capillaire rétinien, qui assure la vascularisation directe des couches rétiniennes internes, et le réseau choroïdien, qui assure, quant à lui, la vascularisation de la rétine de façon indirecte, puisqu'il n'y a pas de capillaires dans la rétine externe. L'artère centrale de la rétine dérive primitivement de la carotide interne, suit un trajet intraneural, pour émerger à la papille optique où elle se divise en ses quatre branches terminales:

${ }^{1}$ Les xanthophylles sont des caroténoïdes oxygénés par la présence notamment d’hydroxyles alcooliques ou d'époxydes.

${ }^{2}$ De vision normale. 
A

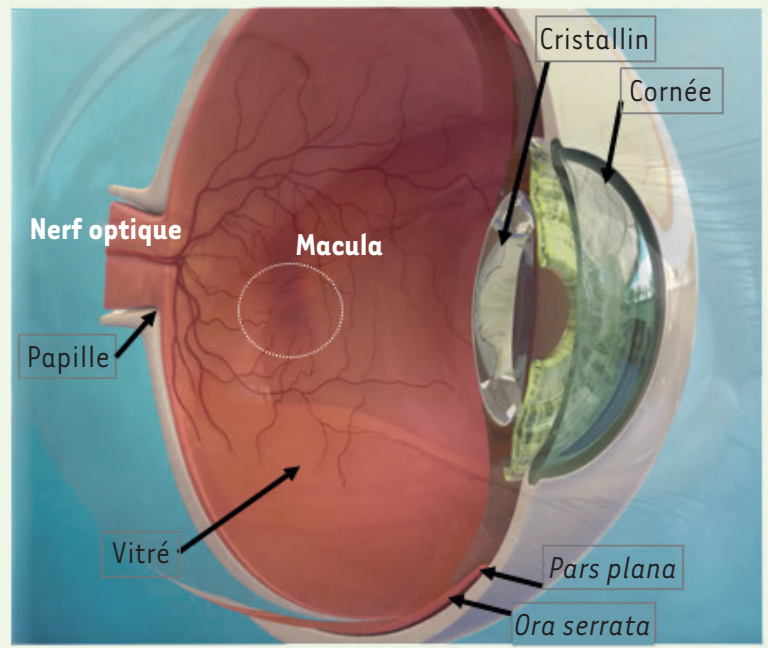

B

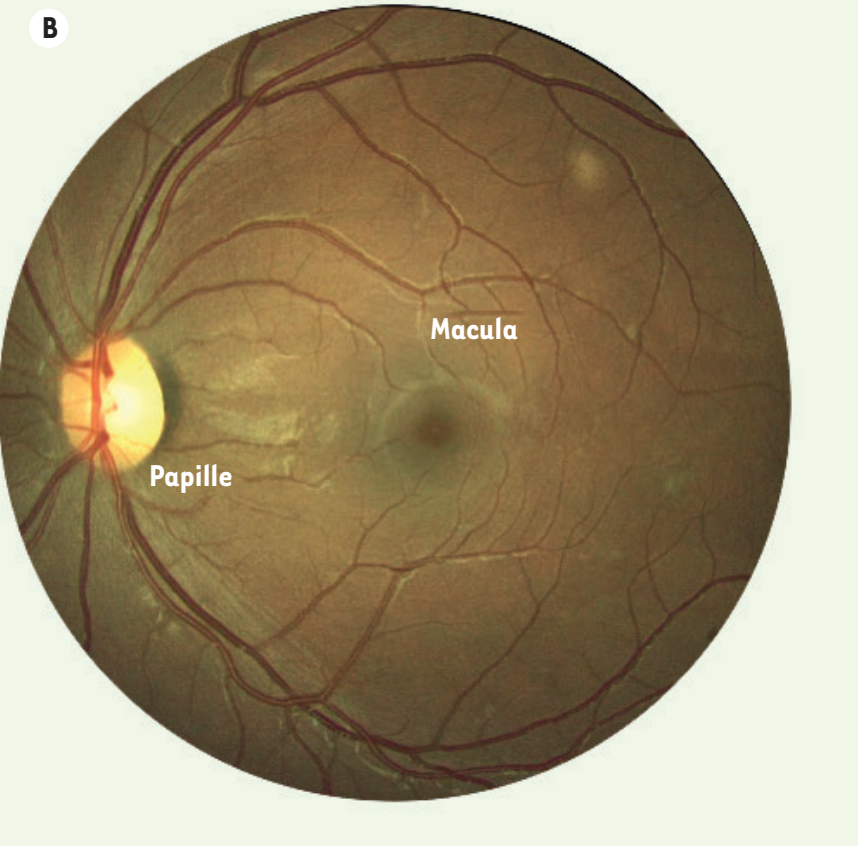

Figure 1. A. Représentation schématique d'un globe oculaire. B. Rétinophotographie couleur d'un fond d'œil humain.

temporales et nasales, supérieures et inférieures. Les artères terminales se divisent en artères collatérales, lesquelles se divisent en artères terminales qui comportent également des collatérales, et ainsi de suite de façon dichotomique jusqu'à former un réseau en grillage couvrant pour chacune des artères, un quadrant de la rétine interne. Les capillaires rétiniens sont issus de ces vaisseaux collatéraux et s'organisent en plexus superficiel, intermédiaire et profond (Figure 2 A, B et Figure 3 C, D).

Les artères et les veines rétiniennes sont localisées dans la couche des fibres optiques. Les capillaires rétiniens ont un diamètre de 5 à $6 \mu \mathrm{m}$ et sont formés de cellules endothéliales à jonctions serrées assises sur une épaisse membrane basale. Des péricytes et des cellules microgliales les entourent. Les types de cellules gliales diffèrent dans les plexus vasculaires superficiels et profonds: alors que dans les plexus vasculaires superficiels et intermédiaires, la communication glio-neuro-vasculaire est assurée par les astrocytes et les cellules gliales de Müller, dans le plexus profond, il n'y a pas d'astrocytes périvasculaires et seules les cellules gliales de Müller peuvent assurer, par les canaux potassiques Kir4.I en particulier, le contrôle des taux de potassium péri-vasculaires (Figure 2 A, B) [1]. Ainsi, en cas de défaillance des cellules gliales de Müller, le réseau capillaire profond est plus sensible à une perte de régulation en réponse à l'activité des photorécepteurs. Les méthodes récentes d'imagerie in vivo, qui permettent de visualiser les flux vasculaires, semblent corroborer ces observations fondamentales (Figure 3 C, D).

Le système vasculaire choroïdien apporte les nutriments et l'oxygène à la rétine externe et, en particulier, aux photorécepteurs de façon indirecte, puisque la rétine externe ne comporte pas de réseau capillaire nourricier. La vascularisation choroïdienne provient de branches de l'artère ophtalmique, elle-même branche de l'artère carotide interne.
La choroïde est un tissu de 300 à $500 \mu \mathrm{m}$ d'épaisseur chez l'homme, limité par la membrane de Bruch en avant, et adhérente à la sclère en arrière (Figure $2 \mathrm{~A}$ ). Elle est constituée de cellules pigmentées (les mélanocytes), de mastocytes, de cellules microgliales et de vaisseaux. Le débit sanguin choroïdien est l'un des plus importants de l'organisme. Les vaisseaux de la choriocapillaire, organisée en lobules fonctionnels irrigués par des artérioles indépendantes, sont formés d'une couche de cellules endothéliales à jonctions serrées comportant de larges fenestrations diaphragmées (60 à $90 \mathrm{~nm}$ ), dont on sait aujourd'hui que l'ouverture dépend du vascular endothelial growth factor (VEGF) (Figure $2 \mathrm{~A}$ et Figure 3 G, F) [2], régulant ainsi le passage des protéines et des macromolécules. Ce gradient protéique entre la rétine et la choroïde est essentiel au maintien de la rétine attaché, et à un état de transparence nécessaire à la transmission des photons [3]. La choroïde est richement innervée par des fibres nerveuses sensorielles parasympathiques, sympathiques et trigéminales qui régulent le flux sanguin choroïdien [4].

\section{Anatomie microscopique}

Classiquement, on distingue dix couches de l'extérieur vers l'intérieur du globe oculaire (Figure $2 \mathrm{~A}$ ) :

- la couche des cellules de l'épithélium pigmentaire rétinien ( $(\varepsilon P R)$; 


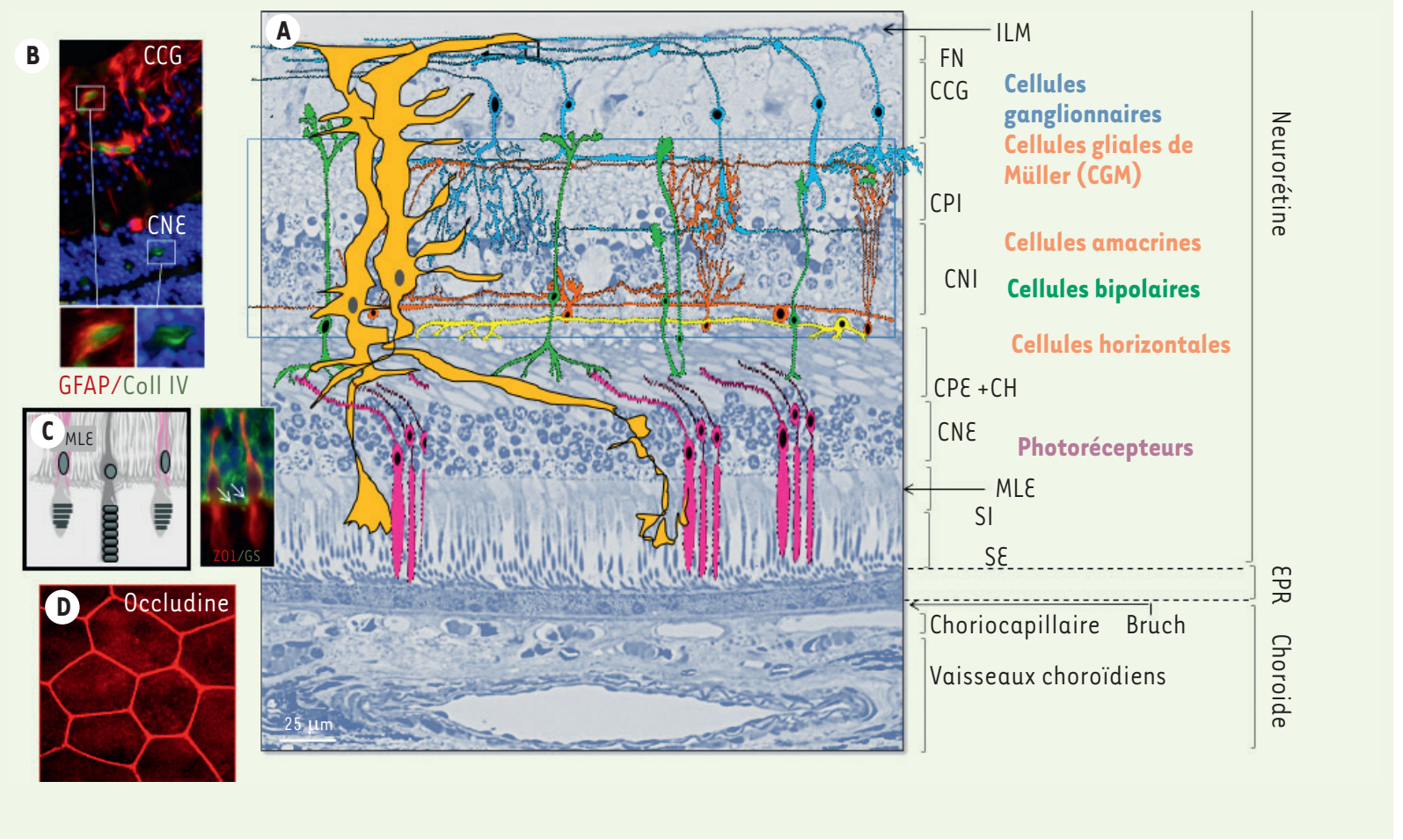

Figure 2. Histologie de la rétine. A. Coupe histologique transversale de rétine humaine au pôle postérieur, ne passant pas par la macula. Les différentes couches sont représentées. Une représentation schématique colorée se superpose pour mettre en évidence les différents types de cellules neuronales et gliales. B. Coupe avec marquage immuno-histochimique des cellules astrocytaires (marquées via la GFAP [green fibrillary acidic protein], rouge) et des parois vasculaires (marquées via le collagène IV, vert), les noyaux sont bleus (DAPI). Les capillaires de la couche profonde ne sont pas entourés d'astrocytes, qui ne couvrent que les vaisseaux des couches superficielles et intermédiaires. C. Les cônes et les bâtonnets sont représentés entourés des prolongements des cellules gliales de Müller (CGM), marquées en vert via la glutamine synthétase (GS, vert). On note la présence de la protéine de jonction $Z 01$ (rouge) entre les photorécepteurs et les CGM au niveau de la membrane limitante externe (MLE). D. Les cellules de l'EPR (épithélium pigmentaire rétinien) forment une monocouche de cellules hexagonales, liées par des jonctions serrées marquées par l'occludine (rouge) sur un montage à plat.

- les segments externes et internes des photorécepteurs $(\mathrm{SI})$;

- la membrane limitante externe, lieu de connexion des cellules gliales de Müller avec les segments internes des photorécepteurs et des photorécepteurs entre eux par des systèmes de jonctions adhérentes et serrées (MLE) ; - la couche de noyaux des photorécepteurs ou couche nucléaire externe $(\mathrm{CNE})$;

- la couche plexiforme externe formée par les synapses entre les cellules bipolaires et les photorécepteurs et avec les cellules horizontales. Ces dernières modulent le message nerveux transmis par la voie directe (CPI) ;

- la couche nucléaire interne qui est la couche des noyaux des cellules horizontales, bipolaires, amacrines et des cellules gliales de Müller. La région externe contient les corps cellulaires des cellules horizontales, la partie centrale les noyaux des cellules bipolaires et des cellules gliales de Müller, ainsi que la partie la plus interne les cellules amacrines ( $\mathrm{CNI}$ ) ;

- la couche plexiforme interne constituée par les dendrites des cellules ganglionnaires et les axones des cellules bipolaires (CPE) ;

- la couche des cellules ganglionnaires (CCG) ;

- la couche des fibres nerveuses, constituée par les axones des cellules ganglionnaires, entourés de prolongements gliaux qui forment le nerf optique relié au cerveau (FN);

- la membrane limitante interne qui est une expansion membraneuse composée des pieds internes des cellules gliales de Müller et de leur membrane basale (MLI).

\section{L'épithélium pigmentaire de la rétine}

La monocouche d'épithélium pigmentaire rétinien ( $(E R)$ est un élément majeur de la rétine, non seulement en raison de ses propriétés physiques et fonctionnelles de barrière hémato-rétinienne externe et de ses propriétés optiques, mais surtout en raison de ses nombreuses activités métaboliques. L'EPR est un épithélium jointif à jonctions serrées (Figure 2 D), hautement polarisé, contenant de multiples canaux ioniques et aqueux dans ses faces baso-latérales et apicales, assurant les transports sélectifs entre la neurorétine et la vasculari- 
sation choroïdienne. L'EPR est indispensable à la vision du fait de son activité métabolique dans le cycle des rétinoïdes, mais aussi du fait de la phagocytose et du recyclage des segments externes «usagés » des photorécepteurs. La monocouche d'EPR contient des pigments formés principalement de grains de mélanine et de lipofucsine. Ces grains sont particulièrement réactifs aux photons de courtes longueurs d'onde (bleus) qui induisent la production d'espèces réactives de l'oxygène (ROS), susceptibles de générer un stress oxydatif.

\section{Les photorécepteurs}

Les photorécepteurs sont des cellules neuronales hautement polarisées [5]. Le segment externe du photorécepteur constitue la partie photosensible. Il est formé par des replis de la membrane plasmique, créant un empilement de disques qui sont renouvelés et phagocytés par les cellules de l'EPR. Le segment interne contient la machinerie métabolique et est relié au segment externe par un cil connecteur. La zone dans laquelle se trouvent les mitochondries peut être visualisée par tomographie par cohérence optique ${ }^{3}$. La membrane limitante externe $(M L E)$ correspond à des complexes jonctionnels entre les photorécepteurs et les cellules gliales de Müller (Figure 2 C) [6]. La couche nucléaire externe contient les noyaux des photorécepteurs. La couche plexiforme externe correspond aux synapses établies entre les photorécepteurs et les cellules bipolaires et horizontales, lesquelles forment une couche plus épaisse, la couche des fibres de Henle, dans la région maculaire $(50 \mu \mathrm{m})$ où se trouvent les cellules gliales de Müller, en « $Z$ » $[7]$ (Figure $2 \mathrm{~A}$ ). Ces différentes structures qui forment la rétine externe peuvent être examinées par tomographie par cohérence optique. Elles constituent des marqueurs de l'intégrité de la rétine et de la fonction visuelle.

Les cellules ganglionnaires et la couche des fibres optiques Les cellules ganglionnaires sont des cellules de 10 à $20 \mu \mathrm{m}$ qui forment des synapses, par l'intermédiaire de leurs dendrites, avec les cellules bipolaires et les cellules amacrines, dans la couche plexiforme interne. Les axones des cellules ganglionnaires convergent vers la papille pour former le nerf optique. Ils forment des fibres entrelacées avec des prolongements provenant des cellules gliales de Müller. Les fibres convergent au niveau du nerf optique avec une orientation radiaire, sauf les fibres maculaires qui, elles, forment un réseau rectiligne interpapillomaculaire.

\section{Cellules gliales de la rétine}

La glie rétinienne, comme la glie neuronale dans le système nerveux central, joue de multiples rôles dans l'homéostasie neuronale : transport de nutriments, régulation hydro-ionique, immunomodulation, production de facteurs neurotrophiques. En situation physiologique basale, les astrocytes et la microglie sont localisés dans les couches rétiniennes internes. Les astrocytes sont également situés autour des

${ }^{3}$ La tomographie par cohérence optique ou OCT (optical coherence tomography) est une technique d'imagerie utilisée en ophtalmologie. Cette technique utilise une onde lumineuse pour capturer la lumière diffusée par un matériau et obtenir ainsi des images tridimensionnelles. Sa résolution est de l'ordre de $1 \mu \mathrm{m}$. vaisseaux rétiniens, sous la membrane limitante interne, et sont très nombreux à l'émergence de la tête du nerf optique. Le noyaux des cellules gliales de Müller se trouvent dans la partie médiane de la couche nucléaire interne, mais leurs prolongements cytoplasmiques s'étendent de la membrane limitante interne jusqu'aux segments internes des photorécepteurs (Figure 2 C). II n'est pas exclu que leurs prolongements puissent affronter les microvillosités des cellules de l'EPR. Les cellules de Müller sont les principales cellules gliales de la rétine des vertébrés. Elles constituent un lien anatomique entre les neurones rétiniens et les compartiments avec lesquels ils échangent des molécules, à savoir les vaisseaux rétiniens, le vitré et l'espace sousrétinien (Figure $2 \mathrm{~A}$ ). Elles sont dotées de nombreux canaux ioniques différents, de récepteurs pour des ligands, de molécules de transport transmembranaire et d'enzymes. Elles expriment en particulier la glutamine synthétase, qui convertit les molécules de glutamate internalisées en glutamine. Elles sont responsables du maintien de l'homéostasie du milieu extracellulaire rétinien (ions, eau, neurotransmetteurs et $\mathrm{pH}$ ). Une de leurs principales caractéristiques est la conductance élevée de leur membrane plasmique vis-à-vis du potassium [8]. Elles sont impliquées dans le métabolisme du glucose rétinien, apportant aux neurones lactate et pyruvate pour leur métabolisme oxydatif, ainsi que dans l'élimination des déchets métaboliques.

Les astrocytes de la rétine sont de deux types. D'une part, des cellules deux fois plus longues que larges sont disposées le long des faisceaux de fibres optiques, sans contact vasculaire. D'autre part, une autre population de cellules astrogliales étoilées traverse la couche des fibres optiques et établissent des contacts non spécialisés avec les vaisseaux rétiniens. En conditions physiologiques, les cellules microgliales sont exclusivement localisées autour des vaisseaux rétiniens

\section{La macula humaine}

La macula humaine représente moins de $5 \%$ de la surface rétinienne totale, mais cette petite zone spécialisée, située au centre de l'axe visuel, assure l'essentiel de la vision photopique et des couleurs, ainsi que de l'acuité visuelle (Figure 1 et Figure 3 A). Dans cette région, toutes les couches sont déplacées, laissant au centre de la macula une zone centrale, la fovéa, formée exclusivement de cônes et de cellules gliales de Müller particulières, dont la structure et la fonction seraient différentes de celles des autres cellules de Müller (Figure 3 A, B). II pourrait exister, sur le toit de la fovéa, un autre type de cellules gliales, probablement de type astrocytaire, dont la fonction reste à définir 


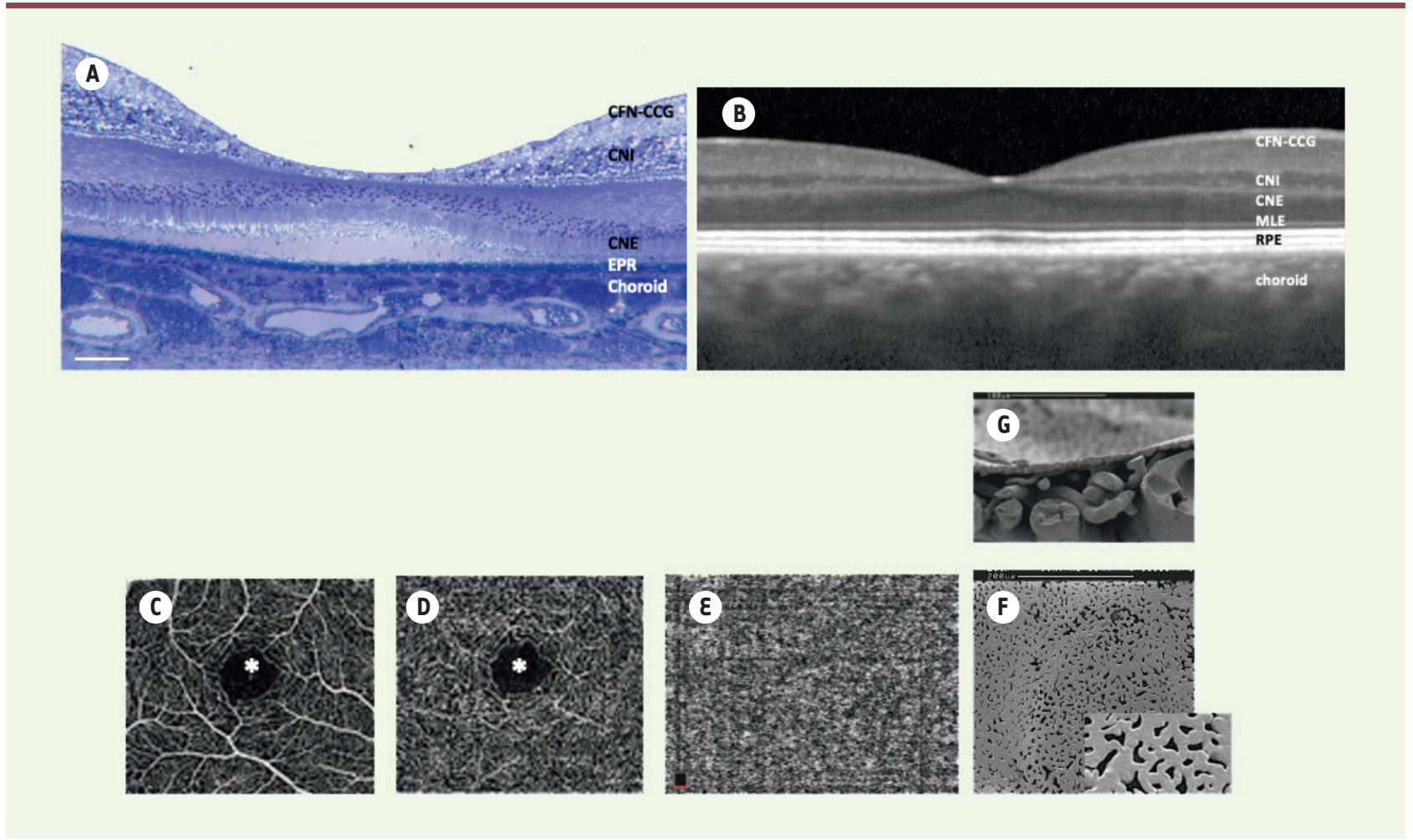

Figure 3. Macula et vascularisation. A. Coupe histologique transversale d'une macula humaine. B. Coupe B-scan en SD-OCT d'une macula humaine normale. $C, D, \varepsilon$. Images de la vascularisation au niveau de la macula, obtenues par OCT-angiographie et permettant sans injection de colorant de visualiser un signal qui témoigne du flux sanguin au niveau de la macula. A : couche des capillaires superficiels; B : couche des capillaires profonds. Noter la zone avasculaire centrale $(*)$. $\varepsilon$ : choriocapillaire; F-G : images de la vascularisation de la choroïde en microscopie électronique à balayage après moulage des vaisseaux par une résine ; F : visualisation de la choriocapillaire de face; $G$ : visualisation de la choriocapillaire et des gros vaisseaux de la choroïde en coupe; CFN : fibres nerveuses ; CCG : couche des cellules ganglionnaires; CNI : couche nucléaire interne ; CNE : couche nucléaire externe; MLE : membrane limitante externe; $\varepsilon P R / R P \varepsilon$ : épithélium pigmentaire de la rétine.

[9]. La densité la plus élevée de cônes se retrouve dans cette zone qui assure l'acuité visuelle, la vision des couleurs et la vision photopique. La macula se développe après la naissance. Elle atteint sa maturité anatomo-fonctionnelle vers 10-12 ans [10]. Seuls les primates non humains et les humains ont une macula, les autres animaux n'en ont pas, exception faite des oiseaux prédateurs, qui, eux, en ont deux ${ }^{4}$. Mais les espèces animales habituellement utilisées dans les laboratoires de recherche en sont dépourvues.

\section{Conclusion}

La structure de la rétine reflète sa complexité fonctionnelle et son extraordinaire spécialisation topographique. De nombreuses analyses structurales, utilisant des méthodes dédiées in vivo et ex vivo, sont encore nécessaires pour, d'une part, préciser la morphologie de la rétine normale humaine et, d'autre part, évaluer les variations physiologiques fonctionnelles. Le recours aux méthodes d'imagerie in vivo ainsi que l'identification de marqueurs spécifiques de différents types

${ }^{4}$ L'acuité visuelle des oiseaux de proie est de loin la plus développée. Elle serait sept à huit fois supérieure à celle de l'homme. cellulaires restent nécessaires pour une analyse morphologique plus précise.

Les méthodes d'imagerie non invasives, comme la tomographie par cohérence optique en domaine spectral (SD-OCT) (Figure $3 B$ ), couplée ou non à une angiographie sans injection de colorant (OCT-A) (Figure $3 C-\varepsilon$ ), ou comme l'optique adaptative ${ }^{5}$, permettent ainsi d'obtenir des images de rétines en coupes transversales (ou «en face»), et même de visualiser certaines cellules. Ces méthodes sont utilisées en pratique courante à des fins diagnostiques et pour guider les décisions thérapeutiques. D’autres méthodes d'imagerie à très haute résolution actuellement en développement, comme la microscopie en contraste de phase par illumination transsclérale ${ }^{6}$,

\footnotetext{
${ }^{5}$ L'optique adaptative est une technologie issue de l'astronomie, qui a pour but d'améliorer les performances d'un système optique en corrigeant les aberrations optiques qu'il induit. Cette technologie permet de visualiser les photorécepteurs de type cône.

${ }^{6}$ Technologie innovante qui permet de visualiser les cellules de l'EPR par contraste de phase obtenu grâce à une illumination oblique transcclérale de la rétine.
} 
permettront d'apporter encore plus de précision pour explorer la rétine normale et pathologique [10]. $\diamond$

\section{SUMMARY}

Anatomy of the retina

The neuroretina is a functional unit of the central nervous system that converts a light signal into a nerve impulse. Of neuroectodermal origin, derived from the diencephalon, the neuroretina is a layered tissue composed of six types of neuronal cells (two types of photoreceptors: cones and rods, horizontal, bipolar, amacrine and ganglion cells) and three types of glial cells (Müller glial cells, astrocytes and microglial cells). The neuroretina lays on the retinal pigmentary epithelium, that together form the retina. The existence of the internal and external blood-retinal barriers and intra-retinal junctions reflects the fineness of regulation of the retinal exchanges with the circulation and within the retina itself. The central zone of the human retina, which is highly specialized for visual acuity, has anatomical specificities. Recent imaging methods make it possible now to enrich our knowledge of the anatomical and functional characteristics of the retina, which are still imperfectly described. $\diamond$

\section{RÉFÉRENCES}

1. Daruich A, Matet A, Moulin A, et al. Mechanisms of macular edema: beyond the surface. Prog Retin Eye Res $2018 ; 63: 20-68$.

2. Cogger VC, Arias IM, Warren A, et al. The response of fenestrations, actin, and caveolin-1 to vascular endothelial growth factor in SK Hepl cells. Am J Physiol Gastrointest Liver Physiol 2008 ; 295 : G137-45.

3. Behar-Cohen F, Dernigoghossian M, Andrieu-Soler C, et al. Potential antiedematous effects of intravitreous anti-VEGF, unrelated to VEGF neutralization. Drug Discov Today 2019 ; 24 : 1436-9.

4. Reiner A, Fitzgerald MEC, Del Mar N, Li C. Neural control of choroidal blood flow. Prog Retin Eye Res 2018; 64 : 96-130.

5. Curcio CA, Sloan KR, Kalina RE, Hendrickson AE. Human photoreceptor topography. J Comp Neurol $1990 ; 292:$ 497-523.

6. Omri S, Omri B, Savoldelli M, et al. The outer limiting membrane (OLM) revisited: clinical implications. Clin Ophthalmol 2010; 4 : 183-95.

7. Matet A, Savastano MC, Rispoli M, et al. En face optical coherence tomography of foveal microstructure in full-thickness macular hole: $a$ model to study perifoveal Müller cells. Am J Ophthalmol 2015 ; 159 : 1142$1151 . e 3$

8. Bringmann A, Pannicke T, Grosche J, et al. Müller cells in the healthy and diseased retina. Prog Retin Eye Res 2006 ; 25 : 397-424.

9. Delaunay K, Khamsy L, Kowalczuk L, et al. Glial cells of the human fovea. Mol Vis $2020 ; 26: 235-45$.

10. Yuodelis $C$, Hendrickson A. A qualitative and quantitative analysis of the human fovea during development. Vision Res $1986 ; 26: 847-55$

\section{LIENS D'INTÉRÊT}

Les auteurs déclarent n'avoir aucun lien d'intérêt concernant les données publiées dans cet article.
TIRÉS À PART

F. Behar-Cohen

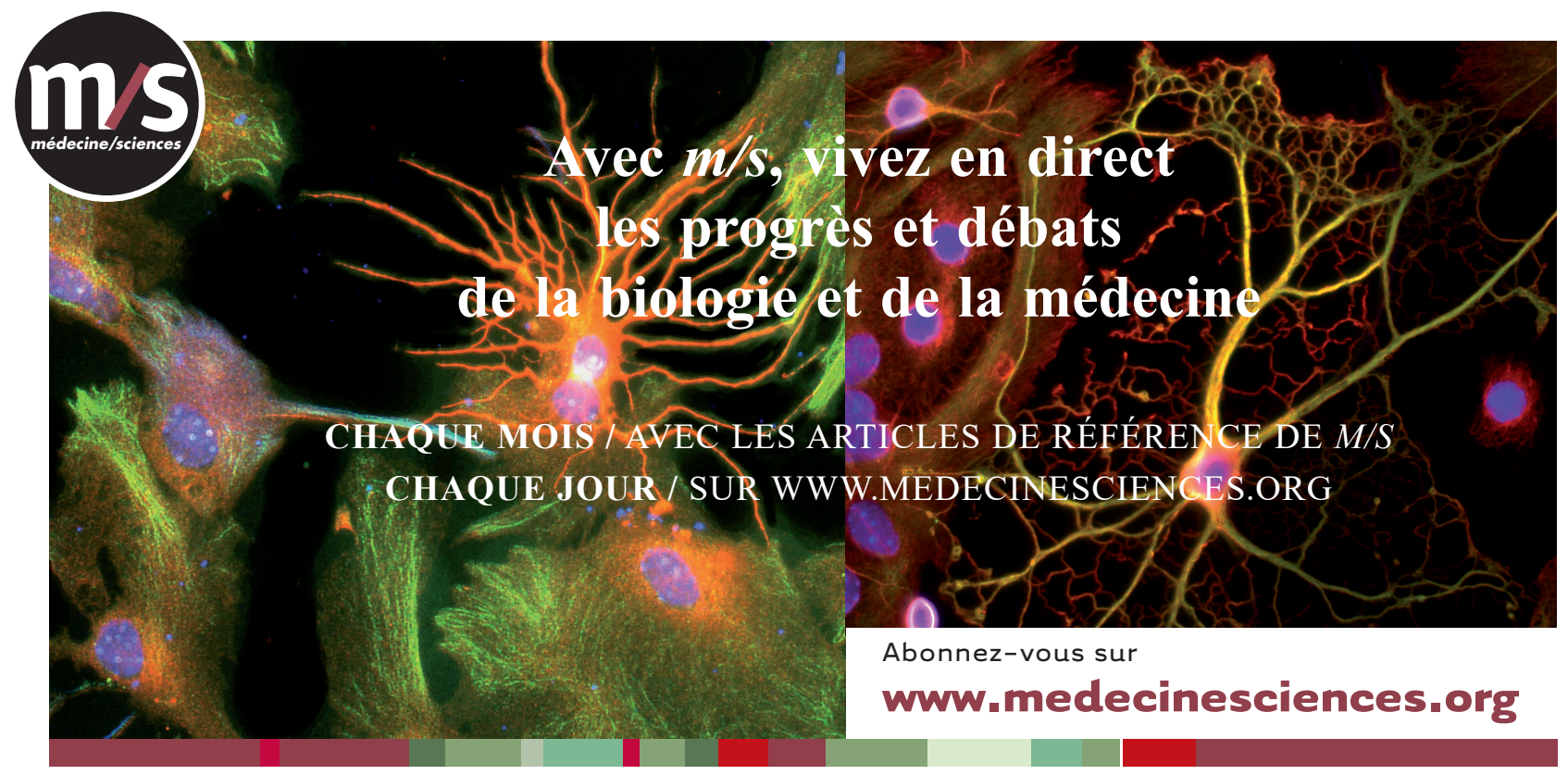

Retrouvez toutes les Actualités de la Myologie sur les sites de :

la Société Française de Myologie

www.sfmyologie.org

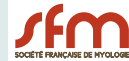

la filière de santé neuromusculaire FILNEMUS

www.filnemus.fr 